\title{
EL PINTOR SEVILLANO JOAQUÍN VALVERDE LASARTE (1896-1982): UNA APROXIMACIÓN A SU VIDA Y A SU OBRA*
}

\author{
SEVILLE PAINTER JOAQUÍN VALVERDE LASARTE \\ (1896-1982): AN APPROACH TO HIS LIFE AND WORK.
}

\author{
Javier García-Luengo Manchado \\ Universidad Isabel I. España \\ Javier_garcia_luengo@yahoo.com
}

\begin{abstract}
Joaquín Valverde Lasarte (Sevilla, 1896-Carmona, 1982), fue un pintor reacio a exposiciones, certámenes o cualquier otra vía de difusión artística, hecho que ha contribuido a un desconocimiento general de su quehacer. Si bien es cierto que algunas investigaciones han abordado la importancia de su trayectoria como pensionado en la Academia de España en Roma, o respecto a los dibujos de una etapa biográfica muy concreta, se hace necesario ofrecer una visión general de su vida y su obra, con el fin de ubicar adecuadamente la producción pictórica de Joaquín Valverde en el devenir artístico español del siglo XX; este es el principal objetivo del presente artículo.

Palabras clave: Retorno al orden, Academia de Roma, Academia de San Fernando.
\end{abstract}

Painter Joaquín Valverde Lasarte (Seville, 1896-Carmona, 1982) was reluctant to exhibitions, contests or any other form of artistic promotion, which contributed to a general lack of knowledge about his endeavor. Although it is true that some research have addressed the importance of his career as pensioner at the Academia de España in Rome, or regarding the drawings of a specific biographical stage, it is necessary to provide a concise overview of his life and work in order to place suitably the pictorial production of Joaquin Valverde through the Spanish art scene of the $20^{\text {th }}$ century. This is the main objective of this article.

Keywords: Return to order, Academy of Spain in Rome, Royal Academy of Fine Arts of San Fernando.

*Deseo dejar constancia de mi más sincera gratitud a los Sres. De Pinaglia Gavira, Valentín Pinaglia Gavira, Francisco Hidalgo Rosendo, Luis Maqueda Toro, Fernando de la Maza Fernández, Jesús Rubio García-Noblejas, Iñigo Ochoa García y a la Fundación Gregorio Prieto, cuyo ánimo e inestimable ayuda han sido fundamentales para llevar a cabo el presente trabajo de investigación. 
La obra del pintor sevillano Joaquín María Valverde Lasarte (Sevilla, 1896-Carmona, 1982), apenas ha sido abordada por la historiografía y la crítica de arte, así como poco divulgada entre el público en general. Ello en buena medida se debe al carácter profesional y humano del propio artista, tímido y concentrado en su continua laboriosidad y en el exigente perfeccionismo de su trabajo. No en vano, fue poco amigo de exposiciones o actividades que de algún modo pudieran difundir su quehacer. Así lo atestiguaba Enrique Lafuente Ferrari, quien a propósito del ingreso de Joaquín Valverde en la Real Academia de Bellas Artes de San Fernando, afirmaría:

“...en el quien se da el raro milagro de haber llegado a la Academia bien doblado el cabo de los cincuenta, sin haber realizado jamás una exposición personal, sin haber acudido, sino raras veces, a exposiciones colectivas, sin haber tocado a rebato las campanas de la crítica ni haber fatigado las prensas que hoy suelen dar aire al nombre de cualquier joven aprendiz...".

En términos similares definiría a Joaquín Valverde su buen amigo, el también pintor, Gregorio Prieto:

"Valverde fue durante toda su vida tan exigente consigo mismo que nunca se permitió hacer una exposición individual de su pintura, llegando a extremos inconcebibles de querer un perfeccionamiento de su propio arte, de tal manera que nunca quedaba contento de su obra, dándola siempre por inacabada y prefiriendo su aislamiento y quizá el silencio de que se ve rodeada su obra y su propia persona".2.

No obstante, la referida ausencia del nombre de Joaquín Valverde Lasarte de las principales publicaciones de historia del arte español contemporáneo, se va restituyendo paulatinamente gracias a diferentes investigaciones que han abordado el estudio de ciertas parcelas biográficas y artísticas del pintor hispalense.

En este sentido cabe destacar al ya citado Enrique Lafuente Ferrari, quien en la también aludida contestación al discurso pronunciado por el pintor sevillano en su ingreso como miembro de número en la Real de Bellas Artes de San Fernando en 1956, ofreció un primer acercamiento sistemático a la trayectoria del personaje que protagoniza el presente artículo ${ }^{3}$.

Hubo que esperar muchos años, prácticamente hasta el fallecimiento del propio pintor, para encontrar alguna referencia rigurosa en este sentido, según

${ }^{1}$ LAFUENTE FERRARI, Enrique: "Contestación del Excmo. Sr. D. Enrique Lafuente Ferrari”, en Discursos leídos ante la Real Academia de Bellas Artes de San Fernando en la recepción pública del pintor Joaquín Valverde Lasarte el 24 de junio. Madrid, 1956, p. 43.

2 PRIETO, Gregorio: "A Joaquín Valverde ese gran artista”, El País, Madrid, 19/01/1983.

${ }^{3}$ LAFUENTE FERRARI, Enrique: "Contestación del Excmo. Sr. D. Enrique Lafuente Ferrari...", op. cit. 
podemos ver en la breve necrológica pronunciada por Pardo Canalís en la Real Academia de Bellas Artes de San Fernando, publicada posteriormente en el Boletín de esta misma institución ${ }^{4}$.

Tiempo después, en 2001, en el contexto universitario, concretamente desde el Departamento de Dibujo I (Dibujo y Grabado) de la Universidad Complutense de Madrid, tuvo lugar la defensa de la tesis doctoral titulada El dibujo en la obra del pintor sevillano Joaquín Valverde, efectuada por José Enrique Pinaglia Gavira, sobrino nieto a la sazón del artista. El grueso de la tesis propone un análisis desde la perspectiva técnica y compositiva de treinta y tres dibujos del autor hispalense, obras conservadas en su mayor parte en la Fundación Rodríguez-Acosta de Granada y que cronológicamente se ubicarían entre 1928 y $1950^{5}$.

La faceta de Valverde como ilustrador, ha sido también analizada en los textos de Juan Manuel Bonet y Fernando Castillo incluidos en el catálogo de la exposición Dos miradas. Una visión: los dibujos de guerra de Carlos Sáenz de Tejada y Joaquín Valverde 6 . Centrándose en las colaboraciones de sendos artistas para la Historia de la Cruzada Nacional, los citados autores dejan a un lado cualquier valoración política en pro de un comedido análisis histórico y estético, para contextualizar y calibrar objetivamente la aportación gráfica tanto de Sáenz de Tejada como de Valverde en el arte español de la pasada centuria.

Cómo no aludir a la exposición comisariada por Adolfo Blanco Osborne en 2003: Roma y la tradición de lo nuevo: diez artistas en el Gianicolo (1923-1927). En dicha muestra se rescataba una de las promociones más sugerentes, pero también más olvidadas de la Academia de España en Roma. Precisamente el texto firmado por Carlos Reyero incluido en el correspondiente catálogo, ofrece una aportación esencial respecto al Valverde de estos años, contextualizándolo con una amplia documentación y objetividad en el devenir estético de la Italia y la Europa de Entreguerras ${ }^{7}$.

Antes de continuar desarrollando alguno de los hitos más importantes de la vida y la obra de Joaquín Valverde, cabe hacer una advertencia respecto a cierta confusión en torno a su origen, pues aunque en muchos casos el gentilicio

${ }^{4}$ PARDO CANALÍS, Enrique: "Necrologías del Excmo. Sr. D. Joaquín Valverde Lasarte”, Academia. Boletín de la Real Academia de Bellas Artes de San Fernando, 55, 1982, pp. 69-74.

5 PINAGLIA GAVIRA, José Enrique: El dibujo en la obra del pintor sevillano Joaquín Valverde. Madrid, 2001. Esta tesis contó con la dirección de Francisco Echauz y Manuel Villaseñor.

${ }^{6}$ BONET, Juan Manuel y CASTILLO, Fernando: Dos miradas. Una visión: los dibujos de guerra de Carlos Sáenz de Tejada y Joaquín Valverde. Madrid, 2010.

7 REYERO, Carlos: Memoria, intertextualidad y una escalera hacia el vacio. La pintura nueva en la Academia y los años veinte en Italia, en Roma y la tradición de lo nuevo: diez artistas en el Gianicolo (1923-1927). Roma, 2003, pp. 36-54. 
carmonense aparece junto a su nombre, lo cierto es que Joaquín Valverde no era natural de Carmona, sino de la cercana Sevilla, donde nació el 21 de agosto de 1896. Sin embargo, tras haber vivido un breve tiempo en la capital andaluza, para marchar junto con su familia más tarde a Córdoba, cuando nuestro pintor contaba tan sólo con 14 años de edad, la tragedia hizo acto de presencia en su familia, al fallecer en poco tiempo su padre y una de sus hermanas, lo que determinaría el traslado de los Valverde Lasarte hasta Carmona, donde existían vínculos familiares maternos ${ }^{8}$.

Es a partir de este momento cuando se produce un singular encuentro entre Valverde y Carmona, tanto desde el punto de vista personal, como estético y por supuesto artístico. Este hecho no es en absoluto casual, para Joaquín Valverde, hombre de refinados gustos, formas y maneras, Carmona suponía una ciudad plena de historia, testimoniada a través del arte y de las importantes colecciones que aquí se atesoran, un lugar donde el misterio y la poesía asoman en cualquiera de sus calles y recoletas plazas, amén de esa intensa luz que generaría un sentido de las formas y del color tan singulares en su quehacer.

A Carmona, a su paisaje y a su paisanaje, Valverde le dedicaría innumerables obras, y aunque bien es verdad que buena parte de su carrera se desarrollaría entre Madrid y Roma, Joaquín pasó largas temporadas en el conocido como Lucero de Andalucía. Por todo ello, aunque Valverde efectivamente no hubiera nacido en Carmona, es considerado como su hijo adoptivo. Fue aquí donde vio por última vez la luz el 22 de diciembre de 1982, siendo enterrado en la Capilla de la Encarnación de la Prioral de Santa María.

Hechas estas acotaciones y centrándonos en la trayectoria del pintor, indudablemente, a tenor del ambiente vivido durante su niñez y sobre todo gracias a la influencia paterna, tan breve como intensa, no es extraño que el joven Valverde sintiera tempranamente una clara vocación artística. Su progenitor, don José Valverde y Ruiz de Somavia, era un gran aficionado a la pintura, de hecho sus descendientes conservan de él algunas marinas de profundo regusto romántico, marinas que en cierto modo rememoraban los años pasados en alta mar por don José, quien había sido oficial de la Armada. Según nos informa José Enrique Pinaglia, probablemente el propio padre del futuro artista fuera quien le enseñara los primeros rudimentos del dibujo y la acuarela a Joaquín ${ }^{9}$.

El afecto por su padre siempre fue intenso. Así, en una carta de Joaquín dirigida a su amigo Gregorio Prieto, le confesaba lo siguiente aludiendo a un viejo retrato fotográfico familiar:

"Me halaga ser el único que asemeja a mi padre en su juventud. Aun reconociendo lo deficiente que es el retrato hay algo que me enternece... me siento íntimamente ligado a

${ }^{8}$ PINAGLIA GAVIRA, José Enrique: El dibujo en la obra ..., op. cit., pp. 20-25.

${ }^{9}$ Ibídem, p. 23. 
una vida de la que sé muy poco. Quisiera haberle escuchado todas las aventuras que después me contaron. Se casó tarde y yo fui el iséptimo! de sus hijos"10.

Por otra parte, la casa familiar de Córdoba, donde vivieron durante casi diez años, la conocida como La Camila, se convirtió en todo un centro de experimentación artística y de debate intelectual. No en vano, los hermanos mayores de nuestro pintor frecuentaban el estudio de Julio Romero de Torres, uno de los creadores más importantes en la España del momento, pintor que por cierto luego sería profesor del propio Valverde ${ }^{11}$.

Así las cosas y dadas las excepcionales dotes de Joaquín para el dibujo, iniciaría su formación en la Escuela Especial de Pintura, Escultura y Grabado de Madrid, denominación oficial de la que popularmente seguía siendo conocida como Escuela de San Fernando. Aprobó la oposición de ingreso en noviembre de 1914 en una convocatoria extraordinaria, ya que por enfermedad no pudo efectuar los ejercicios ordinarios del mes septiembre ${ }^{12}$.

Cursó aquí sus estudios entre 1914 y 1921, llegando a obtener el título de Profesor de Dibujo, título que posteriormente le permitiría dedicarse a la docencia en esta misma casa.

Fueron los compañeros de promoción más destacados, Gregorio Prieto, José Frau, Timoteo Pérez Rubio y Rosa Chacel. La última citada inició su aprendizaje en la escultura, para dedicarse después con notable éxito a la literatura. Precisamente es ella quien nos informa de cómo era este grupo de incipientes artistas y sobre todo, por lo que a nosotros nos interesa, de cómo era nuestro joven pintor:

"Valverde era misterioso. [... ] no había en él la menor frivolidad; su reserva y su distinción le definían como procedente de alta clase - de la clase clásica-y, siendo andaluz, compartía un modesto alojamiento en pensión galdosiana con Tim, el pastor extremeño que malamente entraba en el diminutivo de western que le habíamos asignado"13.

Este núcleo de artistas constituyó, desde mi punto de vista, una de las promociones más destacadas de San Fernando en el siglo XX, siendo quizá también una de las más desconocidas ${ }^{14}$. Entre ellos, además de existir una franca camaradería,

${ }^{10}$ AFGP (Archivo de la Fundación Gregorio Prieto), correspondencia con Joaquín Valverde Lasarte, $16 / 18$.

${ }^{11}$ GAVIRA PINAGLIA, José Enrique: El dibujo en la obra..., op. cit., pp. 23 y 24.

12 Así se desprende de la información recogida en su Expediente Académico: AHUC (Archivo Histórico de la Universidad Complutense) 136/06-37, 39. 14-15.

${ }^{13}$ CHACEL, Rosa: Timoteo Pérez Rubio y sus retratos del jardín. Madrid, 1980, pp.

${ }^{14}$ Sobre esta promoción, véase: GARCÍA-LUENGO, Javier: "La promoción de 1915 de la Escuela Especial de Pintura, Escultura y grabado de Madrid: una visión global", Boletín del Seminario de Arte y Arqueología. Arte, 79, 2013, pp. 227-246. 
había semejantes inquietudes, compartiendo actividades comunes, como salir a pintar a algunos parques madrileños, asistían juntos a exposiciones, etcétera. Posteriormente, en todos los citados la modernidad vendría definida, como veremos en el caso de Valverde, por trabajar en una estética derivada del llamado retorno al orden.

En San Fernando las asignaturas cursadas por Joaquín y sus respectivos profesores fueron: Historia de las Bellas Artes, Leopoldo Soler Pérez; Anatomía artística, José Parada; Dibujo del antiguo y ropajes, Miguel Blay; Paisaje, Antonio Muñoz Degrain y Joaquín Sorolla; Teoría de las Bellas Artes, Rafael Doménech; Perspectiva, Manuel María Magallón y Manuel Marín; Colorido y Composición, Alejo Vera y Juan Antonio Benlliure; Pintura decorativa, Enrique Simonet; Teoría estética del color, Cecilio Plá; Grabado en dulce, Carlos Verger; Dibujo del natural, José Moreno Carbonero; Estética de las Bellas Artes, Ramón María del Valle-Inclán; y Ropajes, Julio Romero de Torres ${ }^{15}$. Testimonio de la brillantez de su expediente académico nos la ofrece los múltiples diplomas de primera, accésit, medallas y premios obtenidos, destacando especialmente sus estudios en Colorido y Composición, Teoría de las Bellas Artes, Pintura Decorativa y Paisaje ${ }^{16}$.

¿Cuál era la estética de Joaquín Valverde por entonces? A tenor de lo que nos ha llegado, el artista sevillano estaba tremendamente influido por los postulados del modernismo, como apreciamos en una de sus ilustraciones (h. 1916-1917), custodiada actualmente en el Museo de la Fundación Gregorio Prieto. Se trata de un tipo de dibujo cuya estética estaba tan en boga por entonces en las principales revistas ilustradas, como podía ser La Esfera o Blanco y Negro, para la que colaboraría. Sus líneas quedan delimitadas por el coup de fuet propio del Art Nouveau, mientras que el tono melancólico general y el color entroncaría con el simbolismo, según denota el paisaje que vemos tras la ventana, tan vinculado a los nabis.

De modernista también podríamos tildar el Retrato de Gregorio Prieto (h. 1918), donde el pintor manchego es efigiado como un auténtico dandi. Así se advierte en la elegante pose de la mano, en el rostro ligeramente altivo y en los tonos decadentistas empleados. El fondo, por su parte, presenta claros débitos con la estética finisecular de Anglada Camarasa, autor tremendamente conocido entonces allende nuestras fronteras y por el que Valverde, según confesó en su discurso de ingreso en la Academia de San Fernando, sentía una especial predilección ${ }^{17}$.

15 AHFBAUC (Archivo Histórico de la Facultad de Bellas Artes de la Universidad Complutense), Libro de registros de matrículas. Alumnos matriculados y calificaciones obtenidas (1912-1923), C. 199.

16 AHFBAUC, datos extraídos de las actas de los cursos: 1914-1915, 1915-1916, 19161917, 1917-1918, 1918-1919, 1919-1920 у 1920-1921; C. 191.

17 VALVERDE, Joaquín: "Observaciones sobre la pintura contemporánea”, en Discursos leídos ante la Real Academia de Bellas Artes de San Fernando en la recepción pública del pintor Joaquín Valverde Lasarte el 24 de junio. Madrid, 1956, p. 22. 
Durante sus estudios en San Fernando, Valverde, junto con los citados Gregorio Prieto, José Frau y Timoteo Pérez Rubio, ganó por dos años consecutivos, en 1918 y 1919, las oposiciones que la Cátedra de Paisaje convocaba para pasar el verano en la Residencia de Paisajistas de El Paular. Este grupo fue el encargado de inaugurar dicha experiencia ${ }^{18}$.

El objetivo de El Paular era constituir una colonia de jóvenes pintores que en medio de un paraje como la sierra madrileña, pudieran asimilar la luz y los efectos atmosféricos in situ. Qué duda cabe que dichos parámetros encajaban con los del impresionismo francés o con experiencias como la Escuela de Barbizon, que con cierto retraso se incorporaban a la formación oficial de la Escuela Especial de Madrid. Entre los trabajos allí efectuados por estos jóvenes, que luego eran expuestos públicamente en otoño, existiría un estilo común singularizado por la desmaterialización de las masas, por los toques rápidos de pincel y los colores vivos. En resumen, unas formas deudoras de los postulados impresionistas y postimpresionistas.

Concluido el periodo de formación, Valverde opositó y ganó el conocido Premio de Roma en 1921. Fue nombrado pensionado el 31 de diciembre de ese año, tomando posesión de su plaza en Roma tres meses más tarde, el 21 de marzo de $1922^{19}$.

La undécima promoción de la Academia de España de Roma, a la que pertenecía nuestro pintor, de alguna manera reabría dicha institución, que prácticamente había cesado su actividad a causa de la I Guerra Mundial. Como consecuencia de ello, fueron muchas las penurias vividas en Roma por este grupo, en compensación pudieron prolongar su estancia dos años más, es decir, frente a los cuatro años reglamentarios de este tipo de becas, los pensionados de 1922 disfrutaron de su estancia en el Janículo durante seis ${ }^{20}$.

La referida promoción, que por tanto se ubicaría cronológicamente entre 1922 y 1928, además de por Joaquín Valverde, estuvo conformada, entre otros, por los arquitectos García Mercadal y Adolfo Blanco, por el músico Fernando Remacha y un largo etcétera. Junto a los citados, Valverde se reencontraría con viejos conocidos de San Fernando, como es el caso del escultor Manuel Álvarez

${ }^{18}$ GARCÍA-LUENGO, Javier: "La primera promoción de la Residencia de Paisajistas de El Paular", Boletín de la Real Academia de Bellas Artes de la Purísima Concepción de Valladolid, 47, 2012, pp. 115-124.

19 CASADO ALCALDE, Esteban: "La Academia de España de Roma entre 1900 y 1936" en Roma: mito modernidad y vanguardia. Pintores pensionados de la Academia de España 1900-1936. Roma, 1998, p. 61.

${ }^{20}$ Sobre esta promoción, véase: BLANCO OSBORNE, Adolfo: "Cuando la vida empieza en Roma o el verdadero sentido de la amistad", en Roma y la tradición de lo nuevo... op. cit., pp. 17-35. 
Laviada, uno de sus grandes amigos hasta la prematura muerte del escultor, acaecida en 1958.

Corresponde precisamente a estos años, concretamente a 1923, la ejecución que Laviada hiciera del Busto de Joaquín Valverde; un retrato que no sólo recoge las características físicas del efigiado, sino que se convierte en un auténtico tributo a la personalidad del sevillano, como así lo advirtió tiempo después Lafuente Ferrari al hablar de este bronce:

"Era entonces Valverde un joven pálido y delgado, de esbelta estatura, metido en sí y con aire de contemplar el mundo con ensimismada y callada reverencia de asombro" 21 .

Asimismo coincidió Valverde en el Janículo con el matrimonio formado por Timoteo Pérez Rubio y Rosa Chacel, pareja con la que también le unía, según hemos visto, una estrecha amistad desde sus primeros años de estudio y de quienes efectuaría por ahora un significativo retrato.

Según ha referido Carlos Reyero, desde el punto de vista artístico, la Italia con la que se encontró Joaquín Valverde, será, como en el resto de Europa, esa Italia del retorno al orden, destacando la estética promovida por el Novecento o Valori Plastici, quienes en la pintura del Quattrocento hallaron un arte lejano al preciosismo finisecular, pero también lejano de las deformidades extremas de las primeras vanguardias. Carlo Carrá, Giorgio De Chirico o Gino Severini, rescataron a Piero Della Francesca, a Fra Angelico o Ucello. A partir de este redescubrimiento, la pintura italiana de este periodo desarrollaría un lenguaje rotundo, monumental, de formas esenciales, un arte donde el silencio y la meditación priman por doquier. Todo lo referido encajaba con el natural de Joaquín Valverde, por lo que el sevillano se insertó plenamente en este ambiente, un ambiente, no olvidemos, de plena modernidad entonces. Dicha coincidencia la supo aprovechar el sevillano ventajosamente ${ }^{22}$.

La querencia de nuestro pintor por el Quattrocento la encontramos ya en su primer envío reglamentario, que le obligaba a copiar "uno de los cuadros o frescos que existan en Italia y ofrezcan mayor interés para la historia del Arte, desde principios del siglo XIII al siglo XVI"23. Tal requerimiento le llevó a reproducir un fragmento de la Disputa de Santa Catalina (1492-1494) de los Apartamentos Borgia del Vaticano, obra de Pinturicchio.

Desde este momento se producirá otro cambio sustancial en la trayectoria de Valverde: su atracción por la pintura mural, por su técnica y sus formas,

${ }^{21}$ LAFUENTE FERRARI, Enrique: “Contestación del Excmo. Sr. D. Enrique Lafuente Ferrari...”, op. cit., p. 46.

22 REYERO, Carlos: Memoria, intertextualidad..., op. cit., pp. 48-51.

${ }^{23}$ CASADO ALCALDE, Esteban: La Academia de España en Roma y los pintores de la primera promoción. Madrid, 1987, t. II, p. 1379. 
influencia que también, como veremos, llevaría incluso hasta la pintura de caba1lete. Este hecho implicaría una evolución extraordinaria en su concepción artística, una evolución que marcaría ya definitivamente su madurez, pasando de los colores vivos y de las formas etéreas deudoras aún de la Escuela de El Paular, a unas composiciones mucho más estructuradas, sólidas y geométricas.

Según marcaba el reglamento, los pensionados por figura, donde se ubicaba nuestro pintor, debían viajar tanto dentro como fuera de Italia. Valverde hizo en lo posible buen uso de dichas obligaciones, y digo en la medida de lo posible pues las restricciones económicas le impidieron efectuar los pertinentes viajes por Europa. No sucedió lo mismo con los viajes por Italia. Al poco de su llegada a Roma sabemos que emprendió una ruta por el norte del país, recalando hasta Venecia, ciudad que frecuentaría en muchas ocasiones a lo largo de su vida.

El último de los envíos reglamentarios de Valverde, El molino (h. 1927-1928) -hoy conservado en el Museo Nacional Centro de Arte Reina Sofía-, hacía las veces de trabajo final que compendiaba el aprendizaje y grado de madurez que el becario había alcanzado en Roma. Con esta obra Joaquín se inscribía plenamente en la modernidad europea, esa modernidad que se alineaba con el Novecento o Valori Plastici. Para su ejecución, el artista vivió una larga temporada en Capri, incluso más allá del tiempo reglamentario. Sin embargo, la dirección de la Academia, consciente de la valía de la obra, hizo caso omiso de las condiciones digamos legales al respecto ${ }^{24}$.

Partiendo de un tema que incluso podríamos calificar como costumbrista o anecdótico, Valverde logra convertirlo en categoría. Construye una obra atemporal mediante lo asimilado de la pintura mural del primer renacimiento. De hecho, este óleo, por su perspectiva, composición, rotundidad y misterio se ha relacionado con la obra maestra de Piero Della Francesca, La Flagelación (1455-1460) del Palacio Ducal de Urbino.

Por otro lado, el sentido de esencialidad y geometría tiene algo de Cézanne, pintor también admirado por Valverde. Así lo atestiguan los bocetos conservados, dibujos que muestran un análisis sintético y concienzudo de la realidad de la que parte.

En cualquier caso, El Molino no lo debemos apreciar como un compendio de influencias, sino como un arte tremendamente personal, maduro, trabajado mediante lo aprendido de la historia, un arte que mira al futuro sin desprenderse del pasado.

La referida modernidad y madurez de El Molino, le valió a Valverde la máxima calificación del jurado que debía valorar los trabajos enviados por los pensionados de Roma. Este cuadro también fue galardonado con la Segunda Medalla en la Exposición Nacional de Bellas Artes de 1930.

${ }^{24}$ REYERO, Carlos: Memoria, intertextualidad..., op. cit., pp. 48-51. 
La profunda vocación muralista de Joaquín, hace que cuando tuviera que trabajar la pintura de caballete, aplicase en muchos casos los modos y las técnicas propios de la pintura mural. Tal y como vemos en la Alegoría Campestre (1930) de la Colección del Banco Santander, cuyo tema de por sí nos remite al Renacimiento, pero no como una mera copia, sino como una inspiración profundamente personal. La modernidad viene dada a partir de la firmeza de las formas, del silencio, del dibujo preciso, en definitiva, de su monumentalidad.

El regreso de Joaquín Valverde a España estaría marcado por un momento de eclosión cultural. Recordemos que un año antes de su retorno tuvo lugar aquel ya casi mítico homenaje a Góngora que capitularía a toda una Generación, la del 27, un grupo donde se aglutinaban literatos, creadores e intelectuales de diferentes credos y tendencias, todos ellos marcados por su anhelo de renovación y vanguardia, modernidad que paradójicamente hallaron en la tradición.

Sin embargo, pareja a la intensa vida cultural encontramos la inestabilidad social y política de este periodo, inquietud que Valverde vivió en primera persona, según se desprende de una carta dirigida a Gregorio Prieto firmada el 28 de junio de 1935, donde Joaquín le recomendaba al pintor manchego lo siguiente:

"Gregorio sigue en Londres donde seguramente tienes todas las fantasías bien fundadas, a ver si puedes dar un salto gigantesco. Esto sigue tan birrioso y mal oliente que no merece la pena hablar de lo que me rodea. [...] Procura olvidar de momento este país de mendigos y acuérdate de este mendiguito que te abraza, Quino" ${ }^{25}$.

Ante esta situación son frecuentes las estancias de Joaquín en Carmona y su campiña, donde hallaba un remanso de paz para seguir dedicado a lo que más le gusta, pintar y dibujar. Pero Carmona también es un lugar que le llena de experiencias estéticas y de vigor, especialmente para una persona de salud tan maltrecha como él. En otra misiva destinada a Gregorio Prieto, poco después de su regreso de Roma, afirma en relación a sus estancias en dicha ciudad:

"Precisamente ese campo o naturaleza que tú refieres es mi único refugio. Hasta el punto de que ya me alarma como una predisposición enfermiza. Nunca he sido tan sensible a la belleza de la luz y del sonido como ahora. Me basta asomarme al campo para sentirme conmovido hasta las entrañas apenas me voy adentrando en él”26.

A pesar de la compleja situación descrita, y animado por el éxito señalado gracias a sus envíos de Roma, a la Segunda Medalla alcanzada en la Nacional de Bellas Artes de 1930 y a la Primera Medalla que lograría en la de 1932, Joaquín Valverde ganó el concurso convocado para efectuar la pintura del techo del salón de recepciones del entonces casi recién inaugurado edificio del Ministerio de

\footnotetext{
${ }^{25}$ AFGP, correspondencia con Joaquín Valverde Lasarte, 16/18.

${ }^{26}$ Ibídem.
} 
Instrucción Pública, hoy de Educación y Ciencia, sito en el número 34 de la madrileña calle de Alcalá.

Valverde se enfrentaba a una pintura mural, soporte que tan bien encajaba con sus anhelos artísticos. Se trataba de una obra enjundiosa, de formato ovalado de más de 8 metros de ancho por casi cinco de alto, en la que nuestro pintor invirtió un prolongado tiempo, el trascurrido entre 1932 y 1935.

Afortunadamente los herederos del pintor, la familia Pinaglia Gavira, conserva el cartón de esta obra, hoy cedido al Museo de la Ciudad de Carmona para su exposición. En él apreciamos el meticuloso proceso de creación de Valverde, un trabajo que implicaba horas de dedicación, de análisis de la realidad hasta llegar a sus formas esenciales.

El resultado es el de una composición absolutamente monumental, capaz de generar un ambiente atemporal sin copiar el pasado, tan solo inspirándose en los ideales clásicos ya descritos. Se elude cualquier anecdotismo en pro de la verdad más pura, de ahí esa belleza que no es caduca, esas maneras que trascienden el tiempo y ese mensaje de quien concebía las Bellas Artes como la única vía para el entendimiento de la humanidad.

De alguna manera, esta alegre exaltación de las Bellas Artes, se vio pronto empañada por el estallido de la Guerra Civil, que Joaquín Valverde vivió en Carmona. No lejos de aquí, en Sevilla, se encontraba su antiguo compañero de estudios, Carlos Sáenz de Tejada, dos promociones posteriores a él en San Fernando. Precisamente Tejada fue nombrado a finales de 1938 director artístico de la Historia de la Cruzada Española. Magna obra editorial promovida por Publicaciones Españolas destinada a recoger minuciosamente, desde el punto de vista del franquismo, la historia de la II República y de la Guerra Civil.

Se trataba de una colección de 36 fascículos, compendiados en 8 volúmenes, editados a todo lujo con un abundante aparato gráfico, que se publicaron entre 1939 y $1943^{27}$. La magnitud de tal empresa, implicaba la necesidad de contar con un importante equipo de ilustradores. Para ello Sáenz de Tejada, conocedor de la valía de Valverde respecto al dibujo, no dudó en requerir su presencia. Si bien es cierto que también colaboraron otros dibujantes, fue en el binomio compuesto por Tejada y Valverde sobre el que recayó prácticamente el grueso de las ilustraciones $^{28}$.

Técnicamente los dibujos de Valverde son herederos del ingrismo practicado por el Picasso del periodo neoclásico y que tanta influencia ejerció en la dibujística europea de los años veinte y treinta. La principal característica de esta

${ }_{27}$ ARRARÁS, J. (Dir.): Historia de la Cruzada Nacional. Madrid, 1939-1942, 36 t. en $8 \mathrm{v}$.

${ }^{28}$ Los datos utilizados para el estudio de este trabajo de Joaquín Valverde, han sido extraídos de: BONET, Juan Manuel y CASTILLO, Fernando: Dos miradas. Una visión..., op. cit. 
tendencia es el empleo de una línea resuelta, fluida y clara, lo que implicaba un conocimiento extraordinario del dibujo y un virtuosismo técnico excelente. A esta línea grácil y fluida, Valverde añade un rico contraste de sombras, sin desechar el abigarramiento compositivo cuando el dramatismo de la escena lo requiere.

Sus dibujos poseen una gran capacidad narrativa y en algunos casos cierto toque costumbrista, sin estar en ayunas, por razones obvias, de ciertas influencias de la serie de Los Desastres de la Guerra (1810-1815) de Goya, especialmente en el dramatismo de algunos claroscuros y en determinados recursos compositivos.

Qué duda cabe que la Historia de la Cruzada Nacional tenía una finalidad propagandística, exaltando al vencedor y recriminando al enemigo. Sin embargo, ni los dibujos de Tejada ni los de Valverde caen en el fácil discurso político, no vemos la caricaturización del enemigo. Vencedores y vencidos están dotados de una notable dignidad. Como ha dicho Fernando del Castillo, "aunque la Historia de la Cruzada Española era un instrumento al servicio de la ideología y de la propaganda, su mérito artístico supera con mucho los propósitos que la impulsaron"29.

Prácticamente, coincidiendo con la conclusión de sus colaboraciones para la Historia de la Cruzada Española en 1942, Joaquín Valverde opositó y ganó la Cátedra de Preparatorio de Colorido de la Escuela de Bellas Artes de San Fernando, para cuatro años más tarde alcanzar la Cátedra de Colorido y Composición, Cátedra otrora ocupada por José de Madrazo, Federico de Madrazo, Dióscoro de la Puebla y un ínclito largo etcétera.

Su forma de trabajo, pulcra y perfeccionista, hace que su dedicación a la docencia sea absoluta, centrando su tiempo para la pintura durante los periodos vacacionales que en la mayor parte pasaba en Carmona, pero también viajando por distintas provincias de Castilla, con el fin de hallar nuevos motivos para su tan inspirada como reconcentrada pintura.

No obstante, también le llegaran encargos de importancia, como su colaboración para la Universidad Laboral de Gijón, uno de los proyectos artísticos y sociales más importantes del primer franquismo, en torno al que se aglutinaron los artistas más relevantes de la España de entonces. En un primer momento se le encargó la decoración del techo del teatro de la Universidad. Un trabajo que se avenía a bien con su trayectoria. Sin embargo, una dolencia pulmonar hizo que el encargo no pasara de un boceto inicial, donde Valverde recreaba algunos elementos tomados de su contacto con el mundo clásico.

En cambio, poco tiempo después, en 1948, sí pudo acometer los cartones para los seis colosales mosaicos que debían decorar otras tantas exedras exteriores de la capilla de la Universidad Laboral. Dichos cartones, conservados por la familia Pinaglia Gavira y cedidos actualmente al Museo de la Ciudad de Carmona, nos muestran a ese Valverde que trabajaba el arte murario de un modo absolutamente

${ }^{29}$ CASTILLO, Fernando: "Dos miradas. Una visión: los dibujos de guerra de Carlos Sáenz de Tejada y Joaquín Valverde”, en Dos miradas. Una visión... op cit., p. 22. 
excepcional. Consciente de que estos dibujos eran la base para los mosaicos que llevaría a cabo el taller de Santiago Padrós Elías, Joaquín Valverde dota a cada una de estas figuras de una rotundidad casi escultórica, en consonancia con el ámbito arquitectónico en el que se insertarían. Trabajó las imágenes de los cuatro evangelistas y de dos arcángeles -San Rafael y San Miguel- a través de grandes planos, con el fin de facilitar el traspaso al muro mediante las teselas.

Una vez más, la técnica de Valverde, basada en la esencialidad, la monumentalidad, los gestos rotundos y esos ademanes, como paralizados en el espacio y el tiempo, otorga a estos dibujos esa atemporalidad tan singular de su peculiar visión del arte sacro.

Poco tiempo después, la Real Academia de Bellas Artes de San Fernando reconocería la actividad pictórica de Joaquín Valverde, así como la de su labor docente en el ámbito de las Bellas Artes, siendo propuesto por Julio Moisés, Juan Adsuara y Enrique Lafuente Ferrari para ocupar el sillón vacante de Marceliano Santamaría. Dicha propuesta fue unánimemente acogida por los académicos en la sesión extraordinaria celebrada el 1 de diciembre de 1952. Sin embargo, la frágil salud de Valverde no le permitirá tomar posesión del cargo hasta el 24 de junio de 1956. En el solemne acto de recepción leyó el discurso titulado: Observaciones sobre la pintura contemporánea ${ }^{30}$; un texto donde apreciamos en Valverde a un atento y consumado teórico del arte del siglo XX, un teórico que apostará por el valor ecuánime del pasado y del presente. Por ello este discurso, así como las valoraciones efectuadas en torno a maestros como Sorolla, Anglada Camarasa, Zuloaga, Manzini, Cézanne o Velázquez, ofrecen una nueva perspectiva respecto a la evolución estética del pintor hispalense.

En este mismo acto, Valverde entregó la obra de recepción que protocolariamente corresponde a los artistas que ingresan en la Real Academia. El creador sevillano donó una pintura de un profundo valor sentimental, el Retrato de mi sobrina (h. 1946). Dicho óleo nos pone ante un consumado retratista, actividad tan importante en el quehacer de Valverde. A partir de un personaje concreto, en este caso su sobrina Amparo Gavira Valverde, de ahí el valor emocional referido de este cuadro, Joaquín desarrolla su peculiar capacidad para reflejar no una belleza temporal o pasajera, sino de una belleza trascendente, aquella que es auténticamente moderna por ser verdaderamente clásica. La aplicación de los criterios asimilados de los grandes maestros del renacimiento, el dibujo sintético o la luz tamizada, amén de la elegante pose, no son elementos ajenos para alcanzar esos valores estéticos y espirituales que Valverde entendía como los más sublimes del arte.

Esta obra testimonia su actividad como retratista, especialmente cuando estaba en Carmona, donde sus jóvenes sobrinas -Ángeles, Esperanza y la ya citada

${ }^{30}$ VALVERDE LASARTE, Joaquín: Observaciones sobre la pintura contemporánea..., op. cit. 
Amparo- se convertían en auténticas musas a la hora de plasmar a través del dibujo o la pintura aquellos conceptos artísticos que Valverde asumía como eternos, de ahí la melancolía dimanada de estas efigies.

En enero de 1960, a la edad de 64 años de edad, el pintor sevillano fue nombrado director de la Academia de España en Roma, cubriendo la vacante dejada por el Marqués de Lozoya. Joaquín regresaba aquella institución en la que casi 35 años antes había dado el salto a la madurez pictórica, tal y como reconocía en un escrito de 1925:

"A Italia y principalmente a Roma le debo el haberme trazado una base estética fuerte y sobria [...] nuestro afán de belleza nos salvará siempre de los convencionalismos $y$ de la indiferencia de cierta gente" ${ }^{\prime 1}$.

Hasta 1967, fecha de su jubilación, Joaquín Valverde dejaría su impronta en tan ínclita institución. Las siguientes palabras del escultor Enrique Pérez Comendador ofrecen un buen resumen a este respecto:

"Valverde durante los siete largos años que dirigió la academia se entregó a ella en cuerpo y alma. Con el máximo acierto. Conociendo a fondo su función, atendió no solo a la buena marcha y efectividad del trabajo de los pensionados, sino que cuidándose del decoro de la institución emprendió obras que embellecieron y ennoblecieron la biblioteca, los salones, comedor de los pensionados, acceso y galerías, sala de exposiciones y cuanto le permitieron los escasos recursos que dispuso.

Conocedor experimentado del espíritu y finalidad de la institución, elaboró además un reglamento en el que colaboramos, junto con López Otero, Director a la sazón de la Real Academia de Bellas Artes de San Fernando",32.

Este regreso a Roma, puso de nuevo en contacto al creador hispalense con aquellos grandes ciclos de pintura religiosa, siempre tan admirados por él. Al hilo de este rencuentro volvió a trabajar la pintura sacra, no era la primera vez que a ello se dedicaba ni mucho menos, recordemos su intervención en la Universidad Laboral de Gijón.

Los lienzos de temática religiosa que el sevillano ejecuta en este momento se caracterizarán por su extraordinario tamaño, pues es en este formato donde Valverde mejor podía recrear los parámetros de la pintura mural. Sin embargo, su obra sacra no está en ayunas de lo que sucedía en la renovación pictórica

${ }^{31}$ Carta de Joaquín Valverde dirigida a Gregorio Prieto, sin fechar, pero por las alusiones debió ser remitida en torno a 1925: A Italia y principalmente a Roma le debo el haberme trazado una base estética fuerte y sobria (...) nuestro afán de belleza nos salvará siempre de los convencionalismos y de la indiferencia de cierta gente. AFGP, correspondencia con Joaquín Valverde Lasarte, 16/18.

32 PARDO CANALÍS, Enrique: Necrologías..., op. cit., p. 73. 
española por entonces. Así en Mensajera del cielo ${ }^{33}$ (h. 1960-1970), su peculiar visión de Santa Teresa de Jesús, o en Cristo en Getsemaní (h. 1960-1970) -ambas propiedad de la familia Pinaglia Gavira-, hallamos un color tremendamente personal aplicado con abundante materia pictórica. Son evidentes los ecos de la Escuela de Madrid y del llamado por Gaya Nuño fauvismo ibérico, tendencias tan importante en la España de los cincuenta y sesenta. Eso sí, Valverde no se dejará llevar irrefrenablemente por dichas coloraciones, antes al contrario, su concepción del dibujo, de la composición y, sobre todo, el sentido místico de lo representado, reflejo por otra parte de sus propias vivencias espirituales, dan a estos lienzos ese aristotélico punto medio a caballo entre la tradición y la renovación.

Precisamente a tenor de estos cuadros se han referido algunos términos que ya para entonces se habían consolidado como invariables en la estética del sevillano: solidez, misterio, espiritualidad, belleza... Curiosamente todo ello lo podemos apreciar en el Autorretrato (h. 1960-1970) de la colección Pinaglia Gavira, auténtico testamento artístico de Valverde, quien no en vano establece un paralelismo entre su gesto meditabundo y la actitud del Velázquez de Las Meninas, con las connotaciones que ello sugiere. El carácter reflexivo que dimana toda la obra, no solo por el ademán, sino también por la esencialidad, la paleta austera y el silencio casi místico, nos pone ante su personal concepción de la actividad creativa, entendida como un auténtico ejercicio de introspección espiritual, cuyo fruto más preciado es la belleza. Quizá este fue el sentido último del arte de Joaquín Valverde Lasarte y, por ende, el fin último de toda una vida consagrada a la pintura.

Fecha de recepción: 28 de septiembre de 2015.

Fecha de aceptación: 20 de noviembre de 2015.

${ }^{33}$ Esta imagen, expuesta en el XXXI Salón de Otoño de Sevilla, sirvió a la prensa para referenciar la Medalla de Honor que la Real Academia de Bellas Artes de Santa Isabel de Hungría de Sevilla concedió en 1982 a Joaquín Valverde Lasarte, poco antes de su muerte. LORENTE, Manuel: "Notable aportación de Joaquín Valverde", $A B C$, Sevilla, 10/11/1982. 


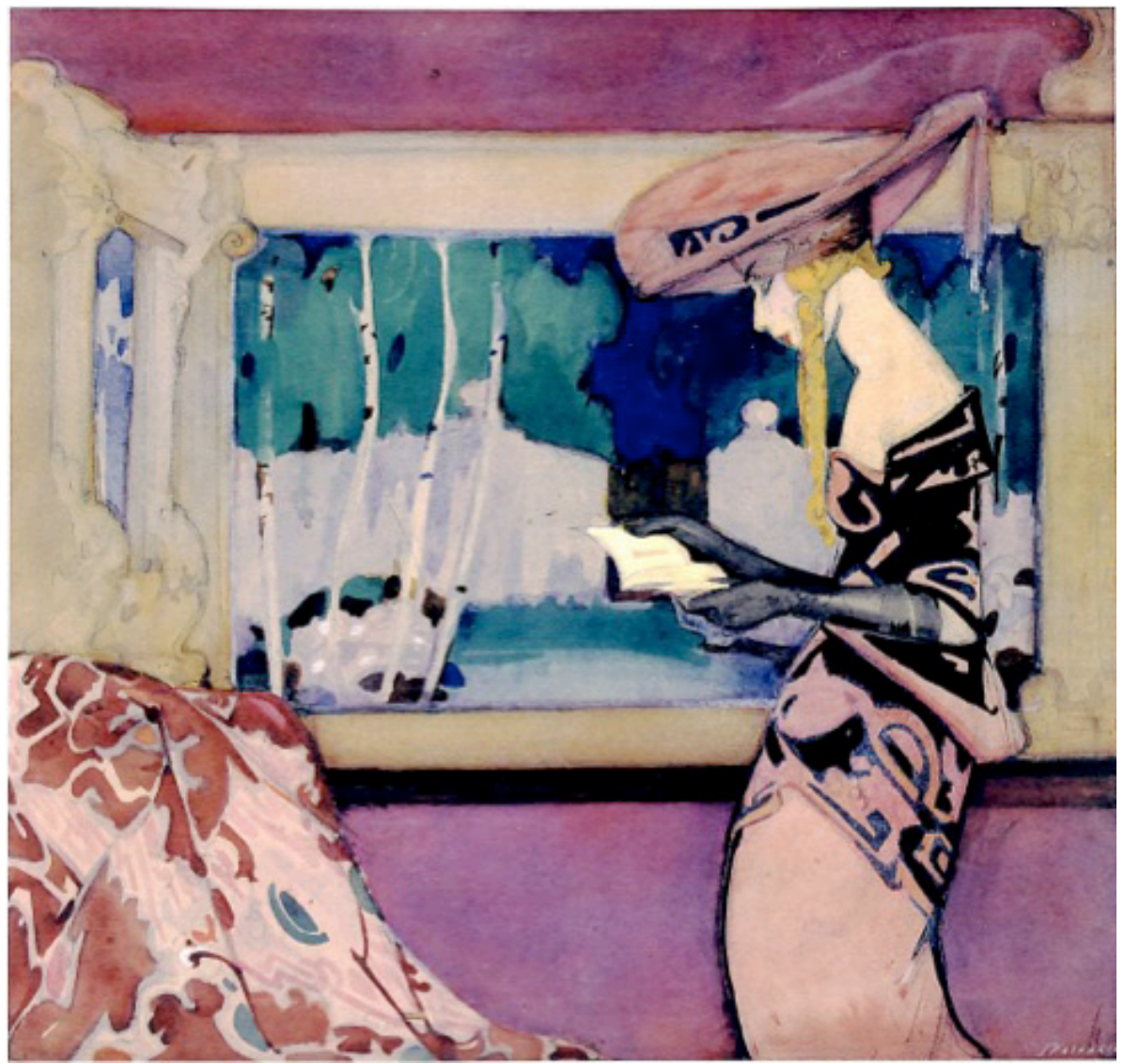

Figura 1. Ilustración. Joaquín Valverde. (H. 1917). Museo de la Fundación Gregorio Prieto, Valdepeñas (Ciudad Real). 


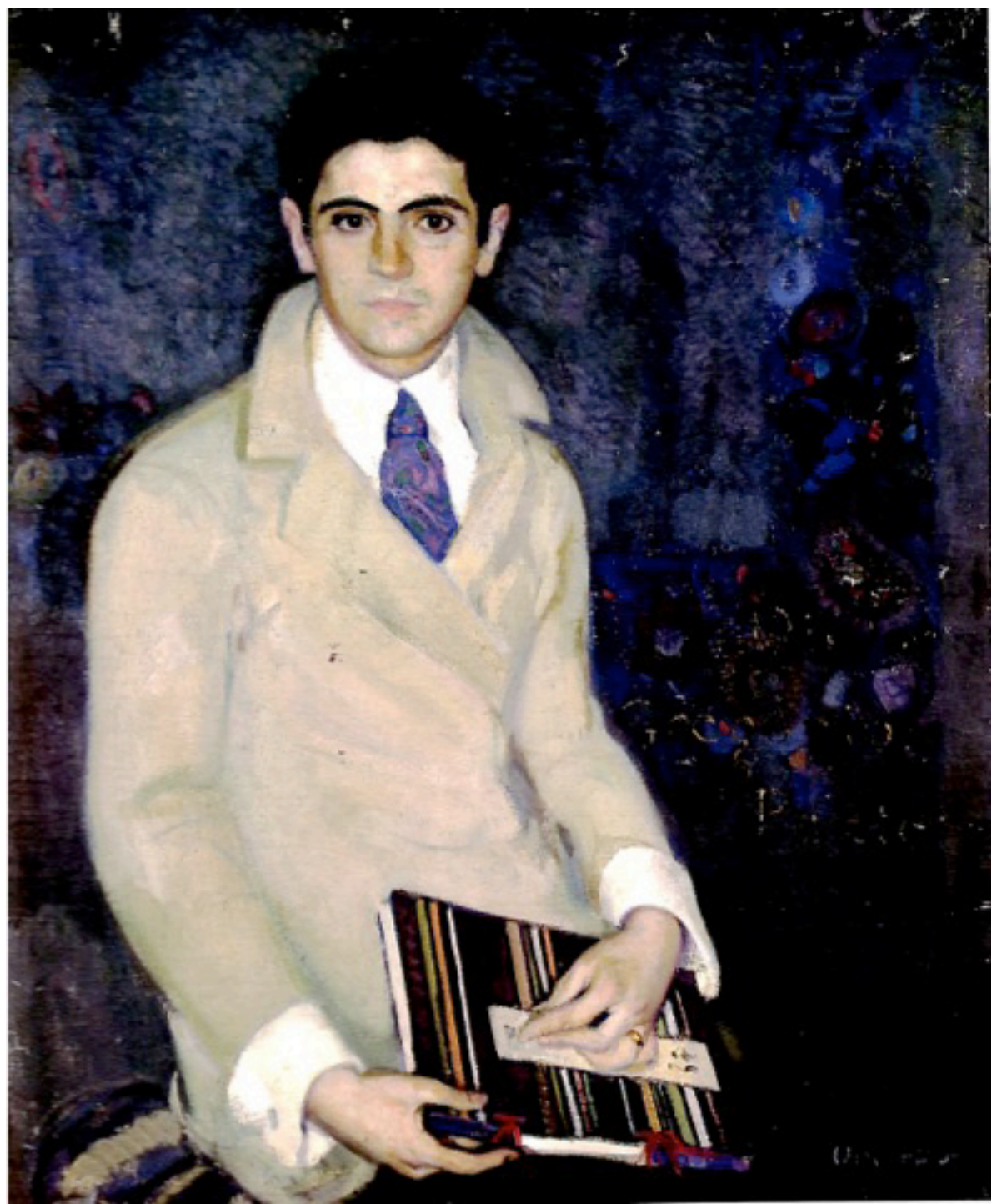

Figura 2. Retrato de Gregorio Prieto. Jaquín Valverde. (H. 1918). Museo de la Fundación Gregorio Prieto, Valdepeñas (Ciudad Real). 


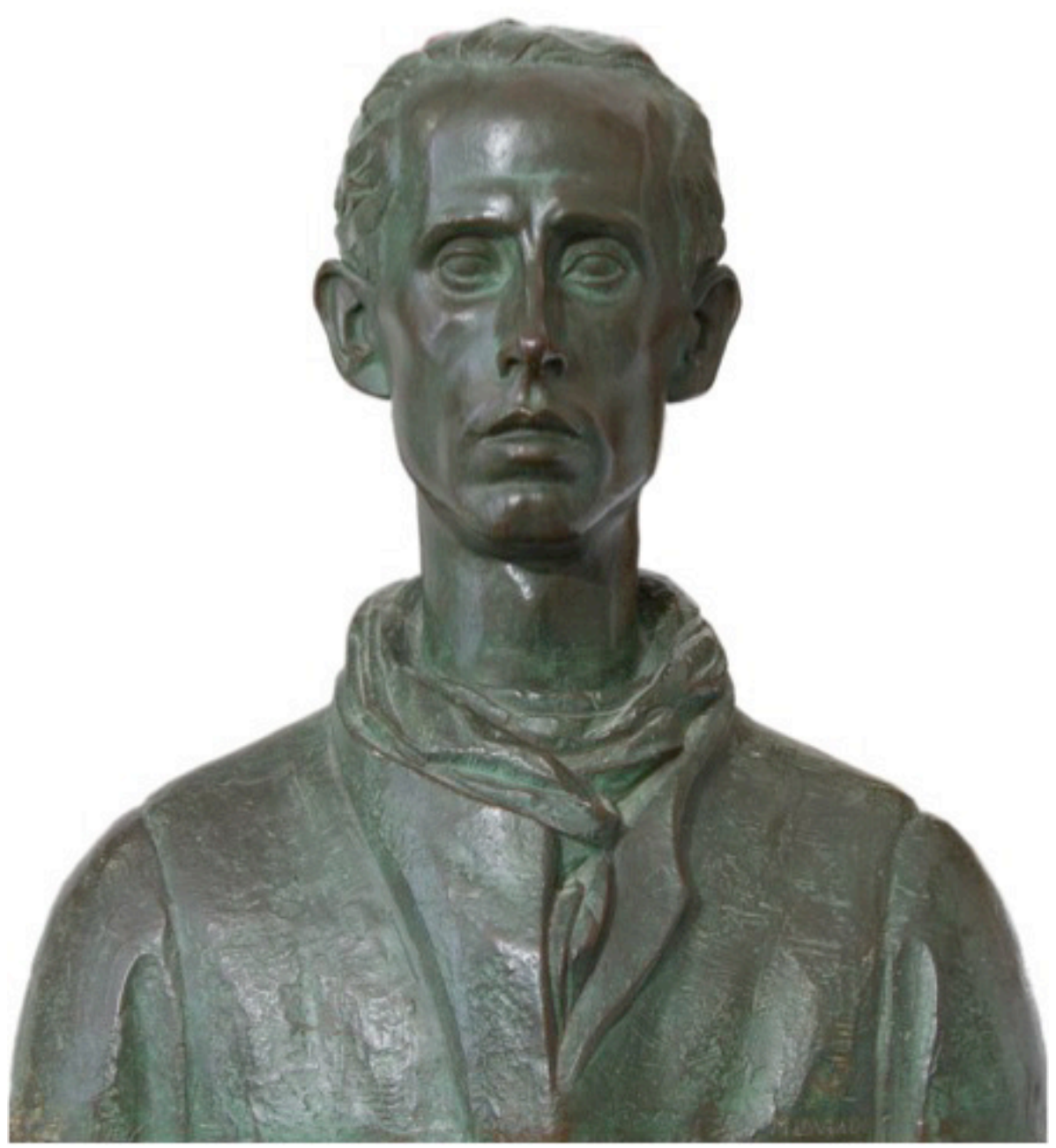

Figura 3. Busto de Joaquín Valverde. Manuel Álvarez Laviada (1923). Colección Pinaglia Gavira, Carmona. 


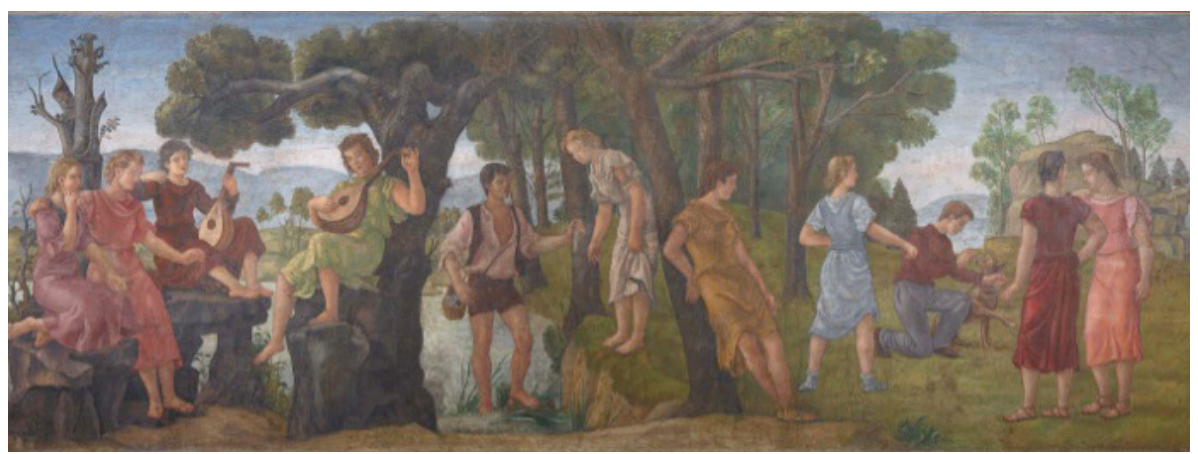

Figura 4. Alegoría campestre. Joaquín Valverde (1930). Colección Banco Santander.

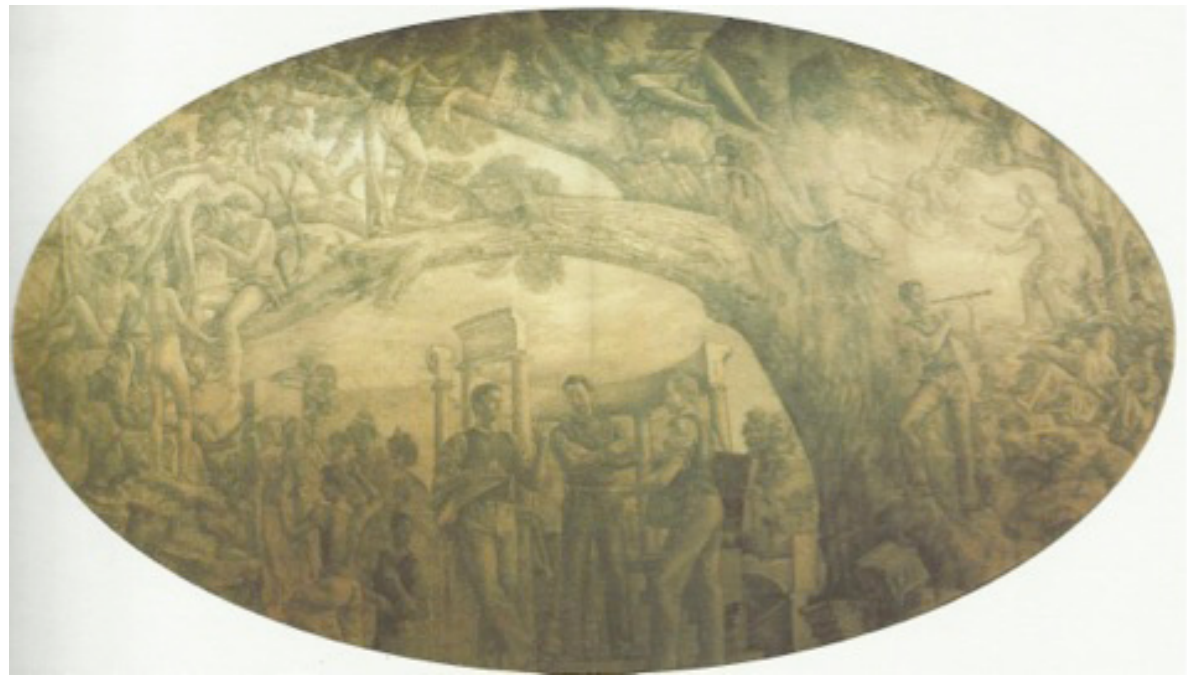

Figura 5. Alegoría de las bellas artes. (H. 1932-1933). Colección Pinaglia Gavira, cedido al Museo de la Ciudad de Carmona. 


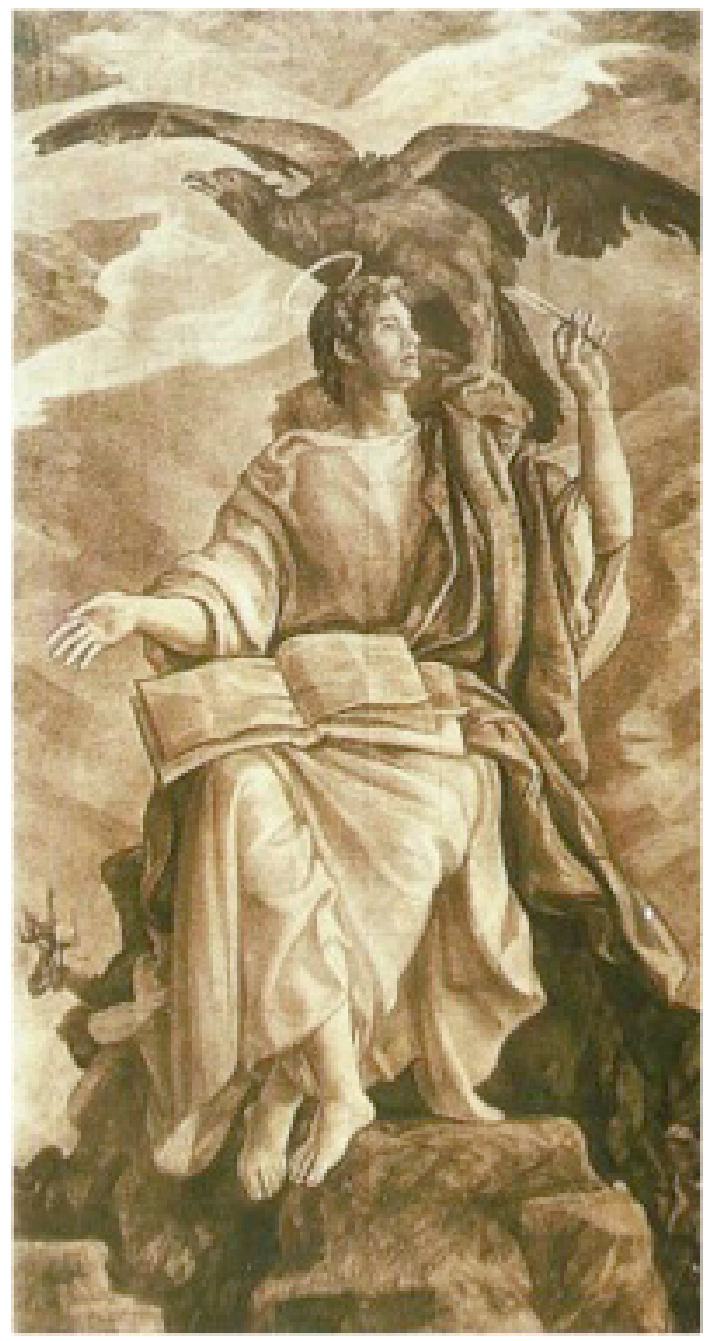

Figura 6. San Juan Evangelista. Joaquín Valverde (H. 1948). Colección Pinaglia Gavira, cedido al Museo de la Ciudad de Carmona. 


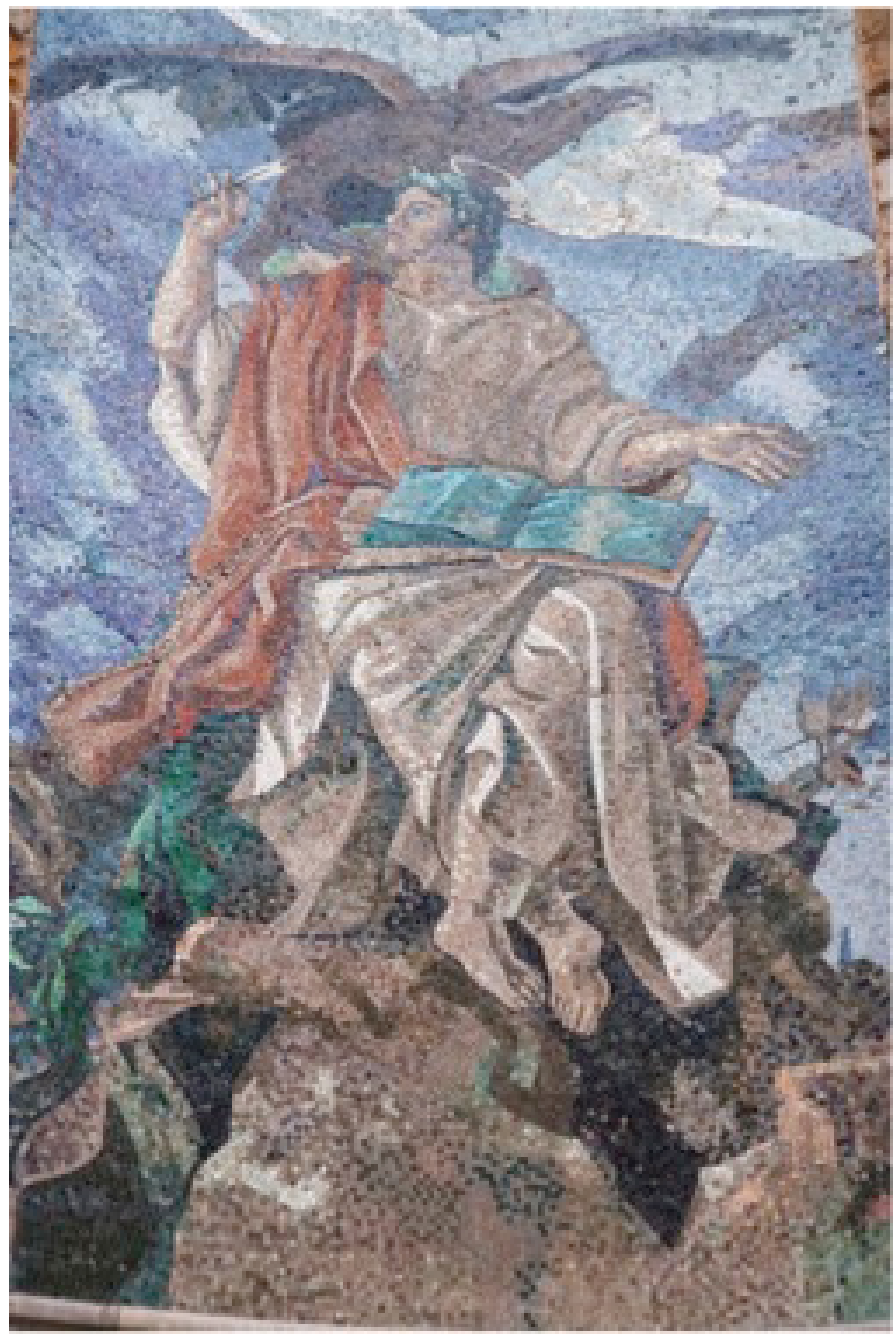

Figura 7. San Juan Evangelista. Taller de Santiago Padrós Elías (1948). Universidad Laboral de Gijón. 


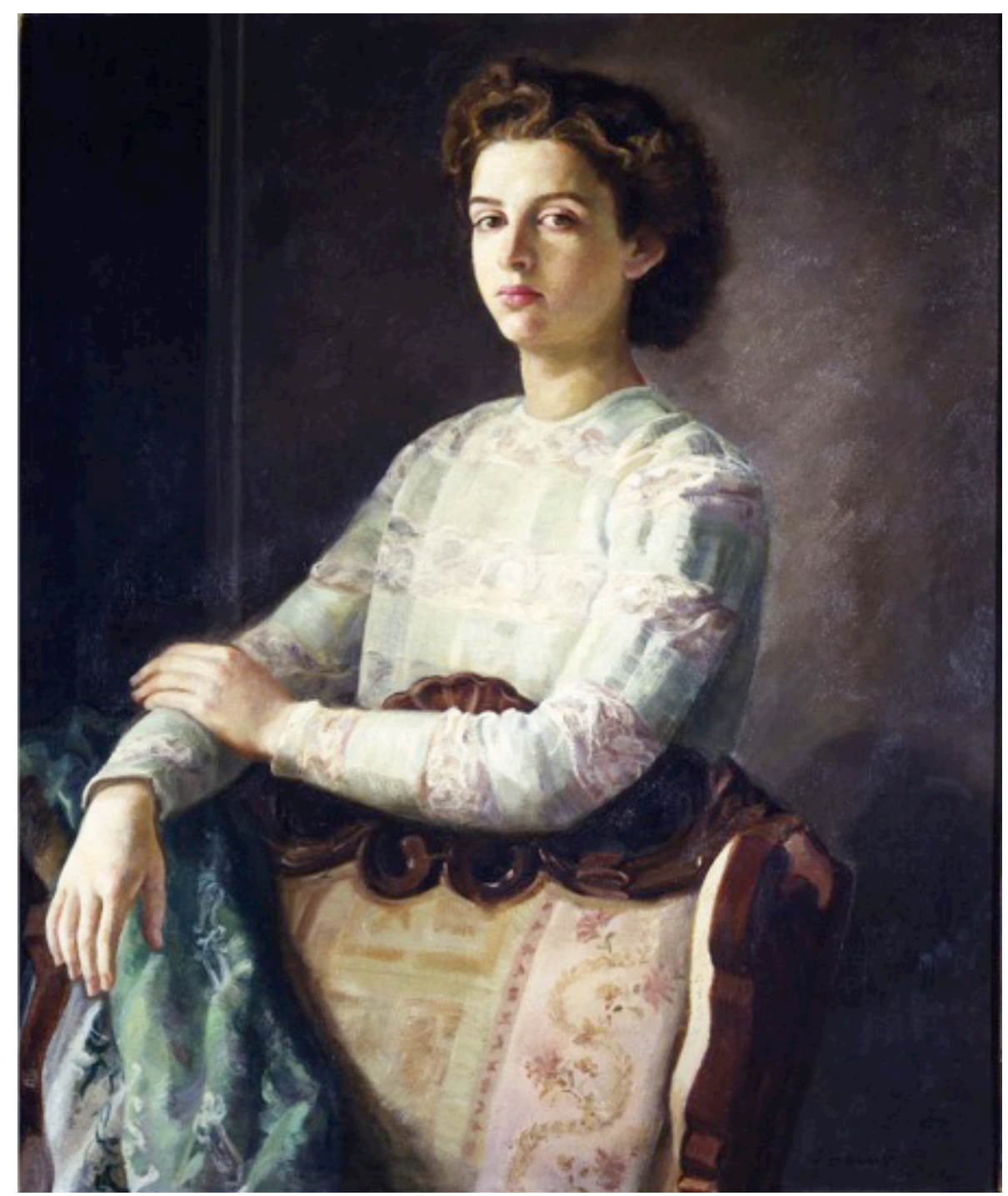

Figura 8. Retrato de mi Sobrina. Joaquín Valverde (H. 1946). Museo de la Real Academia de Bellas Artes de San Fernando, Madrid. 


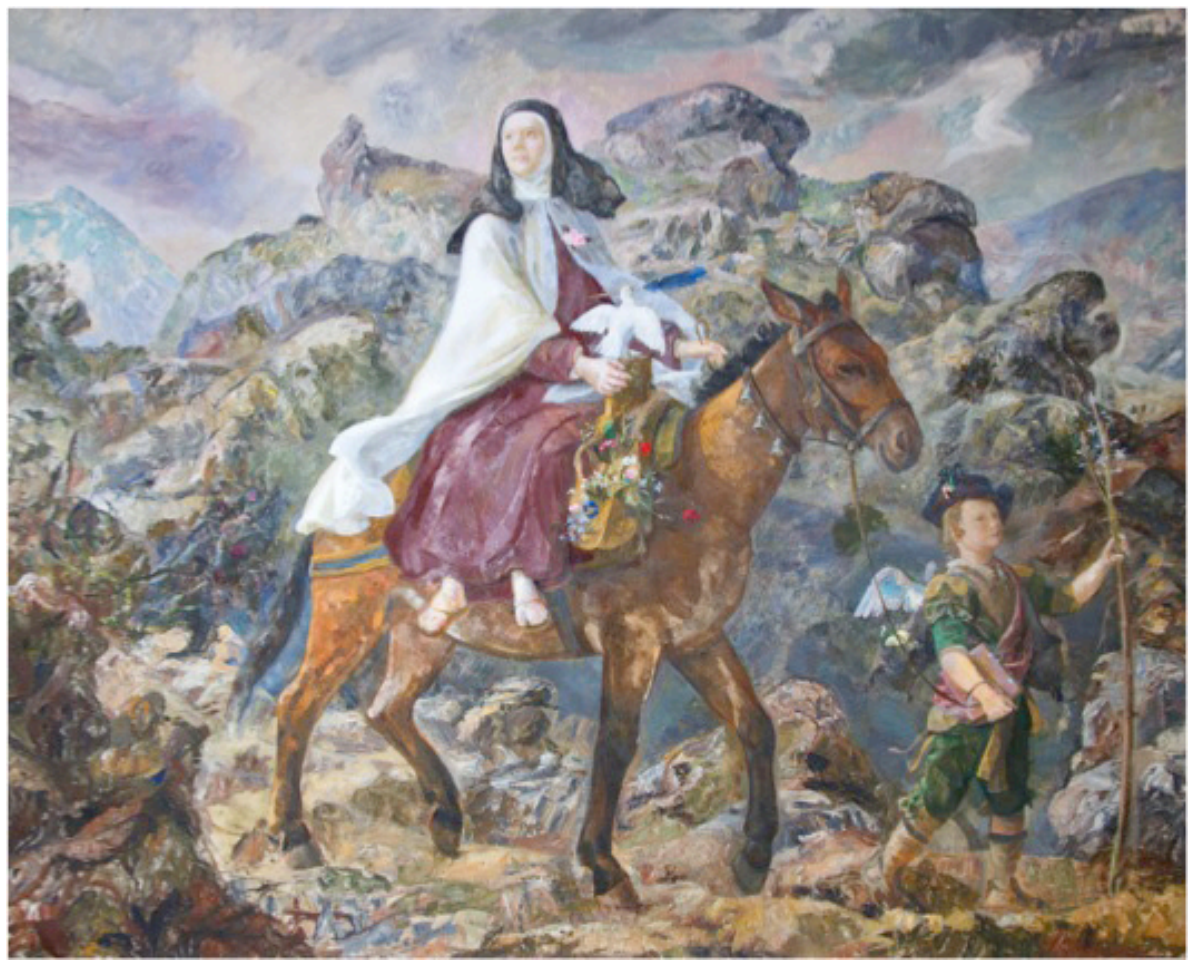

Figura 9. Santa Teresa de Jesús. (H. 1960-1970). Joaquín Vaverde. Colección Pinaglia Gavira, Carmona. 


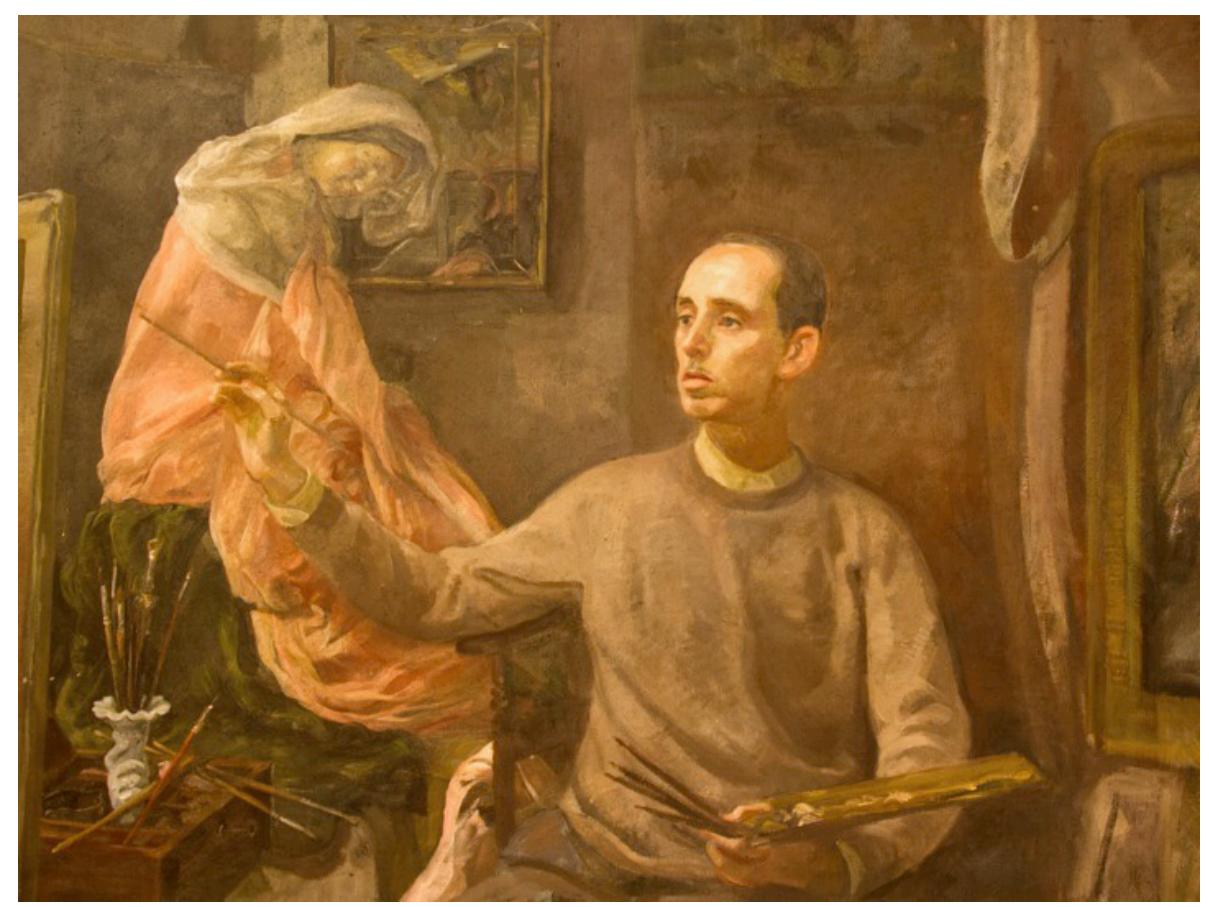

Figura 10. Autorretrato. (H. 1960-1970). Joaquín Vaverde. Colección Pinaglia Gavira, Carmona. 